\title{
Numerical Investigations of a Class of Biological Models on Unbounded Domain
}

\author{
Qiumei Huang ${ }^{1}$, Dongfang $\mathrm{Li}^{2,3, *}$ and Jiwei Zhang ${ }^{4}$ \\ 1 Beijing Institute for Scientific and Engineering Computing, Beijing University of \\ Technology, Beijing 100124, China \\ 2 School of Mathematics and Statistics, Huazhong University of Science and \\ Technology, Wuhan 430074, China \\ ${ }^{3}$ Hubei Key Laboratory of Engineering Modeling and Scientific Computing, Huazhong \\ University of Science and Technology, Wuhan 430074, China \\ 4 Beijing Computational Science Research Center, Beijing, 1000193, China
}

Received 7 October 2017; Accepted (in revised version) 18 January 2018

\begin{abstract}
This paper is concerned with numerical computations of a class of biological models on unbounded spatial domains. To overcome the unboundedness of spatial domain, we first construct efficient local absorbing boundary conditions (LABCs) to reformulate the Cauchy problem into an initial-boundary value (IBV) problem. After that, we construct a linearized finite difference scheme for the reduced IVB problem, and provide the corresponding error estimates and stability analysis. The delay-dependent dynamical properties on the Nicholson's blowflies equation and the Mackey-Glass equation are numerically investigated. Finally, numerical examples are given to demonstrate the efficiency of our LABCs and theoretical results of the numerical scheme.
\end{abstract}

AMS subject classifications: 34A45, 65M12, 65N12

Key words: Biological models, artificial boundary method, absorbing boundary conditions, finite difference method, stability, convergence.

\section{Introduction}

We consider the numerical computation of model equations with delay term on the unbounded spatial domain given as

$$
u_{t}=u_{x x}-d u+f(u(x, t-\tau)), \quad x \in \mathbb{R}, \quad t \in(0, T],
$$

where the initial value is supposed to be compactly supported

$$
u(x, t)=u_{0}(x, t), \quad(x, t) \in \mathbb{R} \times[-\tau, 0] .
$$

${ }^{*}$ Corresponding author. Email address: dfli@hust.edu.cn (D. F. Li) 
Model (1.1) was first proposed to demonstrate the distribution of the Australian blowflies, where $u(x, t)$ represents the mature population of the blowflies, $d>0$ denotes the death rate of the mature population, the delay term $\tau$ represents the time required for a newborn to become matured, the diffusion is used to describe the spatial movement of substances from high to low concentration, and $f(u)$ is the birth-rate function. Two typical birth-rate functions are given as

$$
\begin{aligned}
& f(u)=p u e^{-a u}, \\
& f(u)=\frac{p u}{1+u^{2}},
\end{aligned}
$$

where $p$ is the impact of the birth on the immature population. Model (1.1) with the birthrate function (1.2a) is usually called Nicholson's blowflies equation, and with the function (1.2b) called the Mackey-Glass equation. It is remarkable that this kind of delay equations (1.1) are also considered as models in many other applied scientific fields. One can refer to $[1,3,31]$ for details.

PDEs with delay have been extensively studied. Most results in the literature indicate that a delay term has an important impact on the dynamic properties of a system such as stability, dissipativity, chaos etc. For example, Lin et al. [19] showed that the traveling wave changes with the delay term by using the weighted energy method. Huang and Vandewalle [9] studied the delay-dependent stability of continuous and discrete systems. Strogatz [25] considered the nonlinear dynamic behaviors which is related to the delay term. Zou et al. [45] studied the relation between the delay term and oscillatory behaviors in diffusively coupled dynamical networks. Generally, the investigation on delay-dependent dynamic properties of a system is believed to be one of the most challengeable work. One important reason is that the exact solutions of these PDEs with delay are difficult to obtain.

For the bounded domain case, there are many studies on the numerical simulation of linear and nonlinear PDEs with delay. Jackiewicz and Zubik-Kowal [11] investigated Chebyshev spectral collocation and waveform relaxation methods. Sun [27] applied the linearized compact difference scheme. Zhang and Zhang [38] solved the parabolic problems with delay by combining the compact finite difference method with different time discretization (see also $[15,18,37,39]$ ). Li and Zhang $[16,17]$ introduced the discontinuous Galerkin methods. Zhang and Xiao [37] proposed the implicit-explicit finite difference methods.

For the unbounded domain case, although the theory of problem (1.1) has been well studied (see $[5,19,23]$ ), the numerical analysis for the problem (1.1) has so far received little attention because the unboundedness of the definition domain usually presents a great numerical difficulty. To deal with the unboundedness, one of the powerful tools is to employ artificial boundary methods (ABMs), see the monograph by [8]. The main idea of ABMs is to limit a bounded computational domain of interest by introducing artificial boundary, then impose suitable absorbing boundary conditions (ABCs) on the artificial boundaries, and finally reformulate the unbounded problem into a bounded problem. As repeatedly shown by different authors both theoretically and experimentally, the overall accuracy and performance of numerical schemes strongly depend on the choice of ABCs 
(see papers, $[4,6,7,29,42])$. Up to now, there has been some new progress on designing suitable $\mathrm{ABCs}$ for nonlinear time-dependent problems, such as the linearized or reduced method $([2,12,13,32,35])$, the perfectly matched layer method $([22,28,34])$, the inverse scattering method $([43,44])$ and the unified approach $[20,40,41]$. The other widely used method to deal with the unboundedness is to numerically solve the problem in a sufficiently large domain (see, $[5,19,23]$ ).

In this paper, we consider the derivation of appropriate nonlinear $\mathrm{ABCs}$ for problem (1.1). First, the original problem (1.1) is transformed to a special reaction-diffusion equation with two different terms. After that, the $\mathrm{ABCs}$ are obtained by using the unified approach. Thanks to these ABCs, the problem (1.1) is reduced to an initial-boundaryvalue (IBV) problem. After that, we construct a linearized finite difference method for the reduced IBV problem, and give the corresponding stability analysis and error estimate of the numerical scheme. Numerical examples on the Nicholson's blowflies equation and the Mackey-Glass equation are presented to verify the efficiency of the ABCs and theoretical results of our numerical scheme. We also numerically report that the delay term plays an important role on numerical solutions of the models. As we will see from numerical results, the solutions of Eq. (1.1) behave like a monotone traveling wave when the time delay is small; and the numerical solutions are demonstrated to be chaotically oscillatory when the time delay becomes bigger. These numerical results further demonstrate some of the theoretical findings or conjectures in the references $[5,19,23]$.

The outline of this paper is as follows. In Section 2, we focus on the construction of LABCs. In Section 3, we construct a linearized finite difference scheme for the reduced problem, and establish the stability and convergence of the numerical scheme. In Section 4, numerical examples are given to verify the effectiveness of the theoretical results. Conclusions and discussions are summarized in Section 5.

\section{Construction of nonlinear absorbing boundary conditions}

To derive the efficient ABCs for (1.1), we first recall the general principle of unified approach proposed in $[40,41]$ for the study of the nonlinear Schrödinger equations.

We select two artificial boundaries $\Gamma=\left\{x \mid x=x_{l}, x_{r}\right\}$ such that the initial value $u_{0}$ is compactly supported in the bounded computational domain $\Omega_{i}:=\left\{x \mid x_{l}<x<x_{r}\right\}$. The unbounded exterior domain is defined by $\Omega_{e}=\Omega_{-} \cup \Omega_{+}$with $\Omega_{-}:=\{x \mid-\infty<x<$ $\left.x_{l}\right\}$, and $\Omega_{+}:=\left\{x \mid x_{r}<x<+\infty\right\}$.

\subsection{Unified approach: general principle}

The philosophy of unified approach is given for general equation as follows. Taking $v=u \mathrm{e}^{d t}$, Eq. (1.1) on the exterior domain $\Omega_{e}$ can be equivalently written into

$$
v_{t}=v_{x x}+\mathrm{e}^{d t} g(v(x, t-\tau)), \quad t \in(0, T] .
$$

We further rewrite (2.1) in the operator form as

$$
v_{t}=\mathscr{L} v+\mathscr{N} v,
$$


where $\mathscr{L}$ and $\mathscr{N}$ respectively denote the linear differential operator and the nonlinear operator by

$$
\mathscr{L} v=v_{x x}, \quad \mathscr{N} v=\mathrm{e}^{d t} g(v(x, t-\tau)) .
$$

In analogy to the widely used Strang splitting [24],

$$
v(x, t+\Delta t) \approx e^{\mathscr{L} \Delta t / 2} e^{\mathcal{N} \Delta t} e^{\mathscr{L} \Delta t / 2} v(x, t),
$$

and the first-order approximation (see Baker-Campbell-Hausdroff theorem, [14])

$$
v(x, t+\Delta t) \approx e^{\mathscr{L} \Delta t} e^{\mathscr{N} \Delta t} v(x, t),
$$

in a small time interval from $t$ to $t+\Delta t$ with $\Delta t>0$, we use the approximation as

$$
v(x, t+\Delta t) \approx e^{(\mathscr{L}+\mathscr{N}) \Delta t} v(x, t) .
$$

We first find one-way operator $\mathscr{L}^{(n)}$ to approximate $\mathscr{L}$ by making the wave outgoing. After that, we replace $\mathscr{L}$ by $\mathscr{L}^{(n)}$ in (2.4), then take the limit $\Delta t \rightarrow 0$ of the resulting, finally arrive at the one-way equation

$$
v_{t}=\mathscr{L}^{(n)} v+\mathscr{N} v
$$

The one-way equation (2.5) will be taken as our nonlinear ABCs at artificial boundaries.

Comparing with the well-known time-splitting method (or split-step method), the above procedure is called unified approach. In unified approach, how to obtain a good approximation $\mathscr{L}^{(n)}$ plays an important role. The derivation of the one-way approximate operator $\mathscr{L}^{(n)}$ is considered as follows.

\subsection{Nonlinear absorbing boundary conditions}

To obtain $\mathscr{L}^{(n)}$, we now consider the heat problem on the exterior domain $\Omega_{e}$, namely,

$$
\begin{cases}v_{t}-v_{x x}=0, & x \in \Omega_{e}, \\ v(x, 0)=0, & x \in \Omega_{e}, \\ v \rightarrow 0, & \text { as }|x| \rightarrow \infty .\end{cases}
$$

Applying Laplace transformation to (2.6) with respect to $t$, we arrive at

$$
s \tilde{v}-\tilde{v}_{x x}=0 .
$$

Eq. (2.7) is homogeneous and has the general solution in the form

$$
\tilde{v}(x, s)=A_{1}(s) e^{-\sqrt{s} x}+A_{2}(s) e^{\sqrt{s} x},
$$

where the coefficients $A_{1}(s)$ and $A_{2}(s)$ are arbitrary analytic functions. Using boundary conditions $v(x, s) \rightarrow 0$ when $|x| \rightarrow \infty$ in problem (2.6), and taking derivative with respect to $x$ for (2.8), we arrive at

$$
\frac{\partial \widetilde{v}}{\partial x} \pm \sqrt{s} \tilde{v}=0
$$


The plus sign in " \pm " corresponds to the right boundary, and the minus sign corresponds to the left one. In formula (2.9), we use the Padé approximation to expand the irrational function $\sqrt{s}$ (see [33])

$$
\sqrt{s} \approx \sqrt{s_{0}}-\sqrt{s_{0}} \sum_{k=1}^{N} \frac{b_{k}\left(1-s / s_{0}\right)}{1-a_{k}\left(1-s / s_{0}\right)}
$$

where the parameter $s_{0}$ is the Padé expansion point, and

$$
a_{k}=\cos ^{2}\left(\frac{k \pi}{2 N+1}\right), \quad b_{k}=\frac{2}{2 N+1} \sin ^{2}\left(\frac{k \pi}{2 N+1}\right), \quad k=1,2, \cdots, N .
$$

The higher-order local ABCs and the stability analysis of the resulting boundary conditions are discussed in [33]. Now we consider a simple case $N=1$, namely,

$$
3 s_{0} \widetilde{v}_{x}+s \widetilde{v}_{x} \pm s_{0} \sqrt{s_{0}} \widetilde{v} \pm 3 s \sqrt{s_{0}} \widetilde{v}=0 .
$$

Applying the inverse Laplace transformation, we have

$$
3 s_{0} v_{x}+v_{x t} \pm s_{0} \sqrt{s_{0}} v \pm 3 \sqrt{s_{0}} v_{t}=0 .
$$

We can rewrite (2.12) in form of $\left(\partial_{x} \pm 3 \sqrt{s_{0}}\right) \partial_{t} v=-\left(3 s_{0} \partial_{x} \pm s_{0} \sqrt{s_{0}}\right) v$, which implies that the linear operator $\mathscr{L}$ can be approximated by one-directional operators

$$
\mathscr{L} \approx \mathscr{L}^{(3)}=-\left(\partial_{x} \pm 3 \sqrt{s_{0}}\right)^{-1}\left(3 s_{0} \partial_{x} \pm s_{0} \sqrt{s_{0}}\right) .
$$

Substituting (2.13) into the one-way equation (2.5) with simple calculation, we have the third-order nonlinear local ABCs

$$
\pm 3 \sqrt{s_{0}} v_{t}+3 s_{0} v_{x}+v_{x t} \pm s_{0} \sqrt{s_{0}} v=\left(\partial_{x} \pm 3 \sqrt{s_{0}}\right) e^{d t} g(v(x, t-\tau)) .
$$

Using the definition $v=u e^{d t}$, we can rewrite (2.14) as

$$
\pm 3 \sqrt{s_{0}}\left(u_{t}+d u\right)+3 s_{0} u_{x}+u_{x t}+d u_{x} \pm s_{0} \sqrt{s_{0}} u=\left(\partial_{x} \pm 3 \sqrt{s_{0}}\right) f(u(x, t-\tau)) .
$$

Thus, the problem (1.1) on the real line is reduced to an IBV problem on a bounded computational domain, given by

$$
\begin{array}{lc}
u_{t}=u_{x x}-d u+f(u(x, t-\tau)), & (x, t) \in\left(x_{l}, x_{r}\right) \times \mathbb{R}^{+}, \\
u(x, t)=u_{0}(x, t), & (x, t) \in\left[x_{l}, x_{r}\right] \times[-\tau, 0], \\
u_{x t}-3 \sqrt{s_{0}} u_{t}+\left(3 s_{0}+d\right) u_{x}-\sqrt{s_{0}}\left(3 d+s_{0}\right) u=\left(\partial_{x}-3 \sqrt{s_{0}}\right) f(u(x, t-\tau)), \quad x=x_{l}, \\
u_{x t}+3 \sqrt{s_{0}} u_{t}+\left(3 s_{0}+d\right) u_{x}+\sqrt{s_{0}}\left(3 d+s_{0}\right) u=\left(\partial_{x}+3 \sqrt{s_{0}}\right) f(u(x, t-\tau)), & x=x_{r} .
\end{array}
$$

We remark that the parameter $s_{0}$ is a given positive constant and its choice is not sensitive to the effectiveness of LABCs in this paper. For more discussions, one refers to [36]. 


\section{The fully discrete finite difference method}

In this section, we construct a linearized finite difference scheme for the reduced problem (2.16a)-(2.16d), and give the corresponding stability and convergence analysis.

\subsection{Construction of a finite difference scheme}

Noting that there exist mixed derivatives in the ABCs (2.16c) and (2.16d), it is generally hard to establish their stability analysis. To overcome this difficulty, we introduce two auxiliary variables $\phi(t)=u_{x}\left(x_{l}, t\right)-3 \sqrt{s_{0}} u\left(x_{l}, t\right)$, and $\psi(t)=u_{x}\left(x_{r}, t\right)+3 \sqrt{s_{0}} u\left(x_{r}, t\right)$. Thus, the reduced problem (2.16a)-(2.16d) can be equivalently rewritten as

$$
\begin{array}{ll}
u_{t}=u_{x x}-d u+f(u(x, t-\tau)), & x \in\left(x_{l}, x_{r}\right), 0<t \leq T, \\
u_{x}=\phi(t)+3 \sqrt{s_{0}} u, & x=x_{l}, 0<t \leq T \\
\phi_{t}=\left(\partial_{x}-3 \sqrt{s_{0}}\right) f(u(x, t-\tau))-\left(3 s_{0}+d\right) \phi-8 s_{0} \sqrt{s_{0}} u, & x=x_{l}, 0<t \leq T \\
u_{x}=\psi(t)-3 \sqrt{s_{0}} u, & x=x_{r}, 0<t \leq T \\
\psi_{t}=\left(\partial_{x}+3 \sqrt{s_{0}}\right) f(u(x, t-\tau))-\left(3 s_{0}+d\right) \psi+8 s_{0} \sqrt{s_{0}} u, & x=x_{r}, 0<t \leq T \\
u(x, t)=u_{0}(x, t), & t \leq 0, x \in \Omega_{i} .
\end{array}
$$

Next, we construct the finite difference scheme for the reduced problem (3.1a)-(3.1f).

Let $k=\tau / N_{\tau}$ and $h=\left(x_{r}-x_{l}\right) / M$ be the temporal and spatial step sizes, respectively, where $N_{\tau}$ and $M$ are given positive integers. Denote $t_{n}=n k(0 \leq n \leq N), x_{j}=j h(0 \leq j \leq$ $M), \Omega_{h}=\left\{x_{j} \mid x_{j}=x_{l}+j h, 0 \leq j \leq M\right\}$ and $\Omega_{\tau}=\left\{t_{n} \mid-\tau=t_{-N_{\tau}}<t_{-N_{\tau}+1}<\cdots<t_{N}=T\right\}$ (Without loss of generality, here we assume that there exists a constant $N$ such that $T=$ $N k$ ). Let $\mathscr{V}=\left\{v_{j}^{n} \mid 0 \leq j \leq M, 0 \leq n \leq N\right\}$ be grid function space defined on $\Omega_{h} \times \Omega_{\tau}$. For any grid function $v \in \mathscr{V}$, we will use the following notations

$$
\begin{array}{ll}
\delta_{x} v_{i-\frac{1}{2}}^{n}=\frac{v_{i}^{n}-v_{i-1}^{n}}{h}, & \delta_{x}^{2} v_{i}^{n}=\frac{\delta_{x} v_{i+\frac{1}{2}}^{n}-\delta_{x} v_{i-\frac{1}{2}}^{n}}{h}, \\
\delta_{t} v_{i}^{n+\frac{1}{2}}=\frac{v_{i}^{n+1}-v_{i}^{n}}{k}, & v_{i}^{n+\frac{1}{2}}=\frac{v_{i}^{n}+v_{i}^{n+1}}{2} .
\end{array}
$$

Besides, define the grid functions

$$
u_{i}^{n}=u\left(x_{i}, t_{n}\right), \quad \phi^{n}=\phi\left(t_{n}\right), \quad \psi^{n}=\psi\left(t_{n}\right), \quad 0 \leq i \leq M, \quad-N_{\tau} \leq n \leq N .
$$

Noting that the left boundary point $x_{0}$ holds the equation

$$
u_{x x}\left(x_{0}, t_{n+\frac{1}{2}}\right)=u_{t}\left(x_{0}, t_{n+\frac{1}{2}}\right)+d u\left(x_{0}, t^{n+\frac{1}{2}}\right)-f\left(u\left(x_{0}, t_{n+\frac{1}{2}}-\tau\right)\right),
$$

and using the Taylor expansion, we have

$$
\begin{aligned}
u_{x}\left(x_{0}, t_{n+\frac{1}{2}}\right) & =\frac{1}{h}\left(u\left(x_{1}, t_{n+\frac{1}{2}}\right)-u\left(x_{0}, t_{n+\frac{1}{2}}\right)\right)-\frac{h}{2} u_{x x}\left(x_{0}, t_{n+\frac{1}{2}}\right)+\mathscr{O}\left(h^{2}\right) \\
& =\delta_{x} u_{\frac{1}{2}}^{n+\frac{1}{2}}-\frac{h}{2}\left(\delta_{t} u_{0}^{n+\frac{1}{2}}+d u_{0}^{n+\frac{1}{2}}-f\left(u_{0}^{n+\frac{1}{2}-N_{\tau}}\right)\right)+\mathscr{O}\left(h^{2}\right) .
\end{aligned}
$$


Similarly, at the right boundary point $x_{M}$, we have

$$
u_{x}\left(x_{M}, t_{n+\frac{1}{2}}\right)=\delta_{x} u_{M-\frac{1}{2}}^{n+\frac{1}{2}}+\frac{h}{2}\left(\delta_{t} u_{M}^{n+\frac{1}{2}}+d u_{M}^{n+\frac{1}{2}}-f\left(u_{M}^{n+\frac{1}{2}-N_{\tau}}\right)\right)+\mathscr{O}\left(h^{2}\right) .
$$

Applying the Taylor expansion, (3.2) and (3.3), we have: for $1 \leq n \leq N$

$$
\begin{aligned}
& \delta_{t} u_{i}^{n+\frac{1}{2}}=\delta_{x}^{2} u_{i}^{n+\frac{1}{2}}-d u_{i}^{n+\frac{1}{2}}+f\left(u_{i}^{n+\frac{1}{2}-N_{\tau}}\right)+T_{i}^{n}, \quad 1 \leq i \leq M-1, \\
& \frac{h}{2} \delta_{t} u_{0}^{n+\frac{1}{2}}=\delta_{x} u_{\frac{1}{2}}^{n+\frac{1}{2}}-\phi^{n+\frac{1}{2}}-3 \sqrt{s_{0}} u_{0}^{n+\frac{1}{2}}-\frac{h}{2}\left(d u_{0}^{n+\frac{1}{2}}-f\left(u_{0}^{n+\frac{1}{2}-N_{\tau}}\right)\right)+T_{0}^{n}, \\
& \delta_{t} \phi^{n+\frac{1}{2}}=g\left(u_{0}^{n+\frac{1}{2}-N_{\tau}}\right)-3 \sqrt{s_{0}} f\left(u_{0}^{n+\frac{1}{2}-N_{\tau}}\right)-\left(3 s_{0}+d\right) \phi^{n+\frac{1}{2}}-8 s_{0} \sqrt{s_{0}} u_{0}^{n+\frac{1}{2}}+R_{0}^{n}, \\
& \frac{h}{2} \delta_{t} u_{M}^{n+\frac{1}{2}}=-\delta_{x} u_{M-\frac{1}{2}}^{n+\frac{1}{2}}+\psi^{n+\frac{1}{2}}-3 \sqrt{s_{0}} u_{M}^{n+\frac{1}{2}}-\frac{h}{2}\left(d u_{M}^{n+\frac{1}{2}}-f\left(u_{M}^{n+\frac{1}{2}-N_{\tau}}\right)\right)+T_{M}^{n}, \\
& \delta_{t} \psi^{n+\frac{1}{2}}=g\left(u_{M}^{n+\frac{1}{2}-N_{\tau}}\right)+3 \sqrt{s_{0}} f\left(u_{M}^{n+\frac{1}{2}-N_{\tau}}\right)-\left(3 s_{0}+d\right) \psi^{n+\frac{1}{2}}+8 s_{0} \sqrt{s_{0}} u_{M}^{n+\frac{1}{2}}+R_{M}^{n}, \\
& u_{i}^{j}=u\left(x_{i}, t_{j}\right), \quad i=0,1, \cdots, M, \quad j=-N_{\tau},-N_{\tau}+1, \cdots, 0,
\end{aligned}
$$

where $g(u)=\frac{\partial}{\partial x} f(u)$ and there is a constant $C_{1}$ such that

$$
\begin{array}{lll}
\left|T_{j}^{n}\right| \leq C_{1}\left(k^{2}+h^{2}\right), & 0 \leq j \leq M, & 1 \leq n \leq N, \\
\left|R_{0}^{n}\right| \leq C_{1} k^{2}, & \left|R_{J}^{n}\right| \leq C_{1} k^{2}, & 1 \leq n \leq N .
\end{array}
$$

Omitting the truncation errors, we obtain the finite difference scheme of the problem (3.1a)-(3.1f), for $1 \leq n \leq N$

$$
\begin{aligned}
& \delta_{t} U_{i}^{n+\frac{1}{2}}=\delta_{x}^{2} U_{i}^{n+\frac{1}{2}}-d U_{i}^{n+\frac{1}{2}}+f\left(U_{i}^{n+\frac{1}{2}-N_{\tau}}\right), \quad 1 \leq i \leq M-1, \\
& \frac{h}{2} \delta_{t} U_{0}^{n+\frac{1}{2}}=\delta_{x} U_{\frac{1}{2}}^{n+\frac{1}{2}}-\Phi^{n+\frac{1}{2}}-3 \sqrt{s_{0}} U_{0}^{n+\frac{1}{2}}-\frac{h}{2}\left(d U_{0}^{n+\frac{1}{2}}-f\left(U_{0}^{n+\frac{1}{2}-N_{\tau}}\right)\right), \\
& \delta_{t} \Phi^{n+\frac{1}{2}}=g\left(U_{0}^{n+\frac{1}{2}-N_{\tau}}\right)-3 \sqrt{s_{0}} f\left(U_{0}^{n+\frac{1}{2}-N_{\tau}}\right)-\left(3 s_{0}+d\right) \Phi^{n}-8 s_{0} \sqrt{s_{0}} U_{0}^{n+\frac{1}{2}}, \\
& \frac{h}{2} \delta_{t} U_{M}^{n+\frac{1}{2}}=-\delta_{x} U_{M-\frac{1}{2}}^{n+\frac{1}{2}}+\Psi^{n+\frac{1}{2}}-3 \sqrt{s_{0}} U_{M}^{n+\frac{1}{2}}-\frac{h}{2}\left(d U_{M}^{n+\frac{1}{2}}-f\left(U_{M}^{n+\frac{1}{2}-N_{\tau}}\right)\right), \\
& \delta_{t} \Psi^{n+\frac{1}{2}}=g\left(U_{M}^{n+\frac{1}{2}-N_{\tau}}\right)+3 \sqrt{s_{0}} f\left(U_{M}^{n+\frac{1}{2}-N_{\tau}}\right)-\left(3 s_{0}+d\right) \Psi^{n+\frac{1}{2}}+8 s_{0} \sqrt{s_{0}} U_{M}^{n+\frac{1}{2}}, \\
& U_{i}^{j}=u\left(x_{i}, t_{j}\right), \quad i=0,1, \cdots, M, \quad j=-N_{\tau},-N_{\tau}+1, \cdots, 0
\end{aligned}
$$

where $U_{i}^{n+\frac{1}{2}}, \Phi^{n+\frac{1}{2}}$ and $\Psi^{n+\frac{1}{2}}$ are numerical approximations of $u\left(x_{i}, t_{n+\frac{1}{2}}\right), \phi\left(t_{n+\frac{1}{2}}\right)$ and $\psi\left(t_{n+\frac{1}{2}}\right)$, respectively. 


\subsection{Convergence and stability analysis}

For any $u, v \in \Omega_{h}$, we also denote

$$
\begin{array}{ll}
(u, v)=h\left(\frac{1}{2} u_{0} v_{0}+\sum_{i=1}^{M-1} u_{i} v_{i}+\frac{1}{2} u_{M} v_{M}\right), & \|u\|=\sqrt{(u, u)} \\
|v|_{1}=\sqrt{h \sum_{i=1}^{M}\left(\delta_{x}^{2} v_{i-\frac{1}{2}}\right),} & \|v\|_{\infty}=\max _{0 \leq i \leq M}\left|v_{i}\right| .
\end{array}
$$

First, we introduce some lemmas, which will assist in the proof of our main result.

Lemma 3.1. ([26]) For any mesh function $v$, the following inequality holds

$$
\|v\|_{\infty}^{2} \leq \epsilon|v|_{1}^{2}+\left(\frac{1}{\epsilon}+\frac{1}{x_{r}-x_{l}}\right)\|v\|^{2} .
$$

Lemma 3.2. ([10]) Let $k, B$ and $a_{i}, b_{i}, \gamma_{i}$ be nonnegative numbers for all $i \geq 0$. It holds that

$$
a_{n}+k \sum_{i=0}^{n} b_{i} \leq k \sum_{i=0}^{n} \gamma_{i} a_{i}+B
$$

Suppose that $k \gamma_{i}<1$ for all $i$ and set $\sigma_{k}=\left(1-k \gamma_{i}\right)^{-1}$. Then,

$$
a_{n}+\sum_{i=0}^{n} b_{k} \leq B \exp \left(k \sum_{i=0}^{n} \gamma_{i} \sigma_{i}\right)
$$

Lemma 3.3. ([30]) Let $\left\{\omega^{m}\right\}_{m=0}^{N}$ be a sequence of functions in $\Omega_{h}$. Then

$$
k\left|\omega^{n}\right|_{1} \leq 2 k \sum_{m=1}^{n}\left|\omega^{m-\frac{1}{2}}\right|_{1}+k\left|\omega^{0}\right|_{1} \leq 2 \sqrt{T}\left(k \sum_{m=1}^{n}\left|\omega^{m-\frac{1}{2}}\right|_{1}^{2}+k\left|\omega^{0}\right|_{1}^{2}\right)^{1 / 2} .
$$

Define the error grid functions

$$
\begin{aligned}
& e_{j}^{n}=u_{j}^{n}-u_{j}^{n}, \quad 0 \leq j \leq M, \quad 0 \leq n \leq N, \\
& \widetilde{\phi}^{n}=\phi^{n}-\Phi^{n}, \quad \widetilde{\psi}^{n}=\psi^{n}-\Psi^{n}, \\
& K=\max _{1 \leq n \leq N}\left\{\left\|u^{n}\right\|_{\infty}+\left|\phi^{n}\right|+\left|\psi^{n}\right|\right\}+1 .
\end{aligned}
$$

Theorem 3.1. Suppose that the problem (3.1a)-(3.1f) has unique and smooth solutions $\left\{u\left(x_{i}, t_{n}\right), \psi\left(t_{n}\right), \phi\left(t_{n}\right)\right\}$. Then, there exists a constant $C^{*}$, independent of $h$ and $k$, such that for all $n$

$$
\begin{aligned}
& \left\|U^{n}\right\|_{\infty}+\left|\Phi^{n}\right|+\left|\Psi^{n}\right| \leq K, \\
& \left\|e^{n}\right\|+\left|\widetilde{\phi}^{n}\right|+\left|\widetilde{\psi}^{n}\right| \leq C^{*}\left(k^{2}+h^{2}\right),
\end{aligned}
$$

when $k$, $h$ and $k^{-1} h^{2}$ are sufficiently small. 
Proof. Subtracting (3.6a)-(3.6f) from (3.4a)-(3.4f), we obtain the error equations: for $1 \leq n \leq N$

$$
\begin{aligned}
& \delta_{t} e_{i}^{n+\frac{1}{2}}=\delta_{x}^{2} e_{i}^{n+\frac{1}{2}}-d e_{i}^{n+\frac{1}{2}}+F\left(u_{i}^{n+\frac{1}{2}-N_{\tau}}, U_{i}^{n+\frac{1}{2}}\right)+T_{i}^{n}, \quad 1 \leq i \leq M-1, \\
& \frac{h}{2} \delta_{t} e_{0}^{n+\frac{1}{2}}=\delta_{x} e_{\frac{1}{2}}^{n+\frac{1}{2}}-\widetilde{\phi}^{n+\frac{1}{2}}-3 \sqrt{s_{0}} e_{0}^{n+\frac{1}{2}}-\frac{h}{2}\left(d e_{0}^{n+\frac{1}{2}}-F\left(u_{0}^{n+\frac{1}{2}-N_{\tau}}, U_{0}^{n+\frac{1}{2}-N_{\tau}}\right)\right)+T_{0}^{n} \text {, } \\
& \delta_{t} \tilde{\phi}^{n+\frac{1}{2}}=G\left(u_{0}^{n+\frac{1}{2}-N_{\tau}}, U_{0}^{n+\frac{1}{2}-N_{\tau}}\right)-3 \sqrt{s_{0}} F\left(u_{0}^{n+\frac{1}{2}-N_{\tau}}, U_{0}^{n+\frac{1}{2}-N_{\tau}}\right) \\
& -\left(3 s_{0}+d\right) \widetilde{\phi}^{n+\frac{1}{2}}-8 s_{0} \sqrt{s_{0}} e_{0}^{n+\frac{1}{2}}+R_{0}^{n}, \\
& \frac{h}{2} \delta_{t} e_{M}^{n+\frac{1}{2}}=-\delta_{x} e_{M-\frac{1}{2}}^{n+\frac{1}{2}}+\widetilde{\psi}^{n+\frac{1}{2}}-3 \sqrt{s_{0}} e_{M}^{n+\frac{1}{2}}-\frac{h}{2}\left(d e_{M}^{n+\frac{1}{2}}\right. \\
& \left.-F\left(u_{M}^{n+\frac{1}{2}-N_{\tau}}, U_{M}^{n+\frac{1}{2}-N_{\tau}}\right)\right)+T_{M}^{n}, \\
& \delta_{t} \widetilde{\psi}^{n+\frac{1}{2}}=G\left(u_{M}^{n+\frac{1}{2}-N_{\tau}}, U_{M}^{n+\frac{1}{2}-N_{\tau}}\right)+3 \sqrt{s_{0} F}\left(u_{M}^{n+\frac{1}{2}-N_{\tau}}, U_{M}^{n+\frac{1}{2}-N_{\tau}}\right) \\
& -\left(3 s_{0}+d\right) \widetilde{\psi}^{n+\frac{1}{2}}+8 s_{0} \sqrt{s_{0}} e_{M}^{n+\frac{1}{2}}+R_{M}^{n}, \\
& e_{i}^{j}=0, \quad i=0,1, \cdots, M, \quad j=-N_{\tau},-N_{\tau}+1, \cdots, 0 \text {, }
\end{aligned}
$$

where

$$
\begin{aligned}
& F\left(u_{i}^{n+\frac{1}{2}-N_{\tau}}, U_{i}^{n+\frac{1}{2}-N_{\tau}}\right)=f\left(u_{i}^{n+\frac{1}{2}-N_{\tau}}\right)-f\left(U_{i}^{n+\frac{1}{2}-N_{\tau}}\right), \\
& G\left(u_{i}^{n+\frac{1}{2}-N_{\tau}}, U_{i}^{n+\frac{1}{2}-N_{\tau}}\right)=g\left(u_{i}^{n+\frac{1}{2}-N_{\tau}}\right)-g\left(U_{i}^{n+\frac{1}{2}-N_{\tau}}\right) .
\end{aligned}
$$

Multiplying (3.10a) by $h e_{i}^{n+\frac{1}{2}}$, and summing up for $i$ from 1 to $M-1$, we have

$$
\begin{aligned}
& h \sum_{i=1}^{M-1} \delta_{t} e_{i}^{n+\frac{1}{2}} e_{i}^{n+\frac{1}{2}} \\
= & h \sum_{i=1}^{M-1}\left(\delta_{x}^{2} e_{i}^{n+\frac{1}{2}} e_{i}^{n+\frac{1}{2}}-d e_{i}^{n+\frac{1}{2}} e_{i}^{n+\frac{1}{2}}+F\left(u_{i}^{n+\frac{1}{2}-N_{\tau}}, U_{i}^{n+\frac{1}{2}-N_{\tau}}\right) e_{i}^{n+\frac{1}{2}}+T_{i}^{n} e_{i}^{n+\frac{1}{2}}\right) .
\end{aligned}
$$

Multiplying $e_{0}^{n+\frac{1}{2}}, \widetilde{\phi}^{n+\frac{1}{2}}, e_{M}^{n+\frac{1}{2}}$ and $\widetilde{\psi}^{n+\frac{1}{2}}$ on both sides of (3.10b)-(3.10e), respectively, adding the results with the above formula, then using the following summation formula by parts

$$
-h \sum_{j=1}^{M-1}\left(\delta_{x}^{2} e_{i}^{n+\frac{1}{2}}\right) e_{j}^{n+\frac{1}{2}}-e_{0}^{n+\frac{1}{2}} \delta_{x} e_{\frac{1}{2}}^{n+\frac{1}{2}}+e_{M}^{n+\frac{1}{2}} \delta_{x} e_{M-\frac{1}{2}}^{n+\frac{1}{2}}=\left|e^{n+\frac{1}{2}}\right|_{1}^{2},
$$


we have

$$
\begin{aligned}
& \frac{1}{k}\left(\left\|e^{n+1}\right\|^{2}+\left|\widetilde{\phi}^{n+1}\right|^{2}+\left|\widetilde{\psi}^{n+1}\right|^{2}-\left\|e^{n}\right\|^{2}-\left|\widetilde{\phi}^{n}\right|^{2}-\left|\widetilde{\psi}^{n}\right|^{2}\right)+\left|e^{n+\frac{1}{2}}\right|_{1}^{2} \\
& =h \sum_{i=1}^{M-1}\left(-d e_{i}^{n+\frac{1}{2}} e_{i}^{n+\frac{1}{2}}+F\left(u_{i}^{n+\frac{1}{2}-N_{\tau}}, U_{i}^{n+\frac{1}{2}}\right) e_{i}^{n+\frac{1}{2}}+T_{i}^{n} e_{i}^{n+\frac{1}{2}}\right)-\widetilde{\phi}^{n+\frac{1}{2}} e_{0}^{n+\frac{1}{2}}-3 \sqrt{s_{0}}\left|e_{0}^{n+\frac{1}{2}}\right|^{2} \\
& -\frac{h}{2}\left(d e_{0}^{n+\frac{1}{2}}-F\left(u_{0}^{n+\frac{1}{2}-N_{\tau}}, U_{0}^{n+\frac{1}{2}-N_{\tau}}\right)\right) e_{0}^{n+\frac{1}{2}}+T_{0}^{n} e_{0}^{n+\frac{1}{2}}+G\left(u_{0}^{n+\frac{1}{2}-N_{\tau}}, U_{0}^{n+\frac{1}{2}-N_{\tau}}\right) \widetilde{\phi}^{n+\frac{1}{2}} \\
& -3 \sqrt{s_{0}} F\left(u_{0}^{n+\frac{1}{2}-N_{\tau}}, U_{0}^{n+\frac{1}{2}-N_{\tau}}\right) \widetilde{\phi}^{n+\frac{1}{2}}-\left(3 s_{0}+d\right)\left|\widetilde{\phi}^{n+\frac{1}{2}}\right|^{2}-8 s_{0} \sqrt{s_{0}} e_{0}^{n+\frac{1}{2}} \widetilde{\phi}^{n+\frac{1}{2}}+R_{0}^{n} \widetilde{\phi}^{n+\frac{1}{2}} \\
& +\widetilde{\psi}^{n+\frac{1}{2}} e_{M}^{n+\frac{1}{2}}-3 \sqrt{s_{0}}\left|e_{M}^{n+\frac{1}{2}}\right|^{2}-\frac{h}{2}\left(d e_{M}^{n+\frac{1}{2}}-F\left(u_{M}^{n+\frac{1}{2}-N_{\tau}}, U_{M}^{n+\frac{1}{2}-N_{\tau}}\right)\right) e_{M}^{n+\frac{1}{2}}+T_{M}^{n} e_{M}^{n+\frac{1}{2}} \\
& +G\left(u_{M}^{n+\frac{1}{2}-N_{\tau}}, U_{M}^{n+\frac{1}{2}-N_{\tau}}\right) \widetilde{\psi}^{n+\frac{1}{2}}+3 \sqrt{s_{0} F}\left(u_{M}^{n+\frac{1}{2}-N_{\tau}}, U_{M}^{n+\frac{1}{2}-N_{\tau}}\right) \widetilde{\psi}^{n+\frac{1}{2}} \\
& -\left(3 s_{0}+d\right)\left|\widetilde{\psi}^{n+\frac{1}{2}}\right|^{2}+8 s_{0}{\sqrt{s_{0}}}^{n+\frac{1}{2}} \widetilde{\psi}^{n+\frac{1}{2}}+R_{M}^{n} \widetilde{\psi}^{n+\frac{1}{2}} .
\end{aligned}
$$

By (3.5a), (3.5b) and Cauchy-Schwarz inequality, we have

$$
\begin{aligned}
& -\widetilde{\phi}^{n+\frac{1}{2}} e_{0}^{n+\frac{1}{2}}+T_{0}^{n} e_{0}^{n+\frac{1}{2}}-8 s_{0} \sqrt{s_{0}} e_{0}^{n+\frac{1}{2}} \widetilde{\phi}^{n+\frac{1}{2}}-3 \sqrt{s_{0}}\left|e_{0}^{n+\frac{1}{2}}\right|^{2} \\
\leq & \frac{1}{4 \sqrt{s_{0}}}\left|\widetilde{\phi}^{n+\frac{1}{2}}\right|^{2}+\frac{1}{4 \sqrt{s_{0}}}\left|T_{0}^{n}\right|^{2}+16 s_{0}^{2} \sqrt{s_{0}}\left|\widetilde{\phi}^{n+\frac{1}{2}}\right|^{2}+3 \sqrt{s_{0}}\left|e_{0}^{n+\frac{1}{2}}\right|^{2}-3 \sqrt{s_{0}}\left|e_{0}^{n+\frac{1}{2}}\right|^{2} \\
\leq & \left(\frac{1}{4 \sqrt{s_{0}}}+16 s_{0}^{2} \sqrt{s_{0}}\right)\left|\widetilde{\phi}^{n+1}\right|^{2}+\left(\frac{1}{4 \sqrt{s_{0}}}+16 s_{0}^{2} \sqrt{s_{0}}\right)\left|\widetilde{\phi}^{n}\right|^{2}+\frac{C^{*}}{4 \sqrt{s_{0}}}\left(k^{2}+h^{2}\right), \\
& \widetilde{\psi}^{n+\frac{1}{2}} e_{M}^{n+\frac{1}{2}}+T_{M}^{n} e_{M}^{n+\frac{1}{2}}+8 s_{0} \sqrt{s_{0}} e_{M}^{n+\frac{1}{2}}-3 \sqrt{s_{0}}\left|e_{M}^{n+\frac{1}{2}}\right|^{2} \\
\leq & \frac{1}{4 \sqrt{s_{0}}}\left|\widetilde{\psi}^{n+\frac{1}{2}}\right|^{2}+\frac{1}{4 \sqrt{s_{0}}}\left|T_{M}^{n}\right|^{2}+16 s_{0}^{2} \sqrt{s_{0}}\left|\widetilde{\psi}^{n+\frac{1}{2}}\right|^{2}+3 \sqrt{s_{0}}\left|e_{M}^{n+\frac{1}{2}}\right|^{2}-3 \sqrt{s_{0}}\left|e_{M}^{n+\frac{1}{2}}\right|^{2} \\
\leq & \left(\frac{1}{4 \sqrt{s_{0}}}+16 s_{0}^{2} \sqrt{s_{0}}\right)\left|\widetilde{\psi}^{n+1}\right|^{2}+\left(\frac{1}{4 \sqrt{s_{0}}}+16 s_{0}^{2} \sqrt{s_{0}}\right)\left|\widetilde{\psi}^{n}\right|^{2}+\frac{C^{*}}{4 \sqrt{s_{0}}}\left(k^{2}+h^{2}\right) .
\end{aligned}
$$

Substituting (3.12a) and (3.12b) into (3.11), we have

$$
\begin{aligned}
& \quad \frac{1}{k}\left(\left\|e^{n+1}\right\|^{2}+\left|\widetilde{\phi}^{n+1}\right|^{2}+\left|\widetilde{\psi}^{n+1}\right|^{2}-\left\|e^{n}\right\|^{2}-\left|\widetilde{\phi}^{n}\right|^{2}-\left|\widetilde{\psi}^{n}\right|^{2}\right)+\left|e^{n+\frac{1}{2}}\right|_{1}^{2} \\
& \leq h \sum_{i=1}^{M-1} F\left(u_{i}^{n+\frac{1}{2}-N_{\tau}}, U_{i}^{n+\frac{1}{2}-N_{\tau}}\right) e_{i}^{n+\frac{1}{2}}+\frac{h}{2} F\left(u_{0}^{n+\frac{1}{2}-N_{\tau}}, U_{0}^{n+\frac{1}{2}-N_{\tau}}\right) e_{0}^{n+\frac{1}{2}}+\frac{h}{2} F\left(u_{M}^{n+\frac{1}{2}-N_{\tau}}, U_{M}^{n+\frac{1}{2}-N_{\tau}}\right) e_{M}^{n+\frac{1}{2}} \\
& \quad+G\left(u_{0}^{n+\frac{1}{2}-N_{\tau}}, U_{0}^{n+\frac{1}{2}-N_{\tau}}\right) \widetilde{\phi}^{n+\frac{1}{2}}-3 \sqrt{s_{0}} F\left(u_{0}^{n+\frac{1}{2}-N_{\tau}}, U_{0}^{n+\frac{1}{2}-N_{\tau}}\right) \widetilde{\phi}^{n+\frac{1}{2}} \\
& \quad+G\left(u_{M}^{n+\frac{1}{2}-N_{\tau}}, U_{M}^{n+\frac{1}{2}-N_{\tau}}\right) \widetilde{\psi}^{n+\frac{1}{2}}+3 \sqrt{s_{0}} F\left(u_{M}^{n+\frac{1}{2}-N_{\tau}}, U_{M}^{n+\frac{1}{2}-N_{\tau}}\right) \widetilde{\psi}^{n+\frac{1}{2}} \\
& \quad+C_{1}\left|\widetilde{\psi}^{n+1}\right|^{2}+C_{1}\left|\widetilde{\psi}^{n}\right|^{2}+C_{1}\left|\widetilde{\phi}^{n+1}\right|^{2}+C_{1}\left|\widetilde{\phi}^{n}\right|^{2}+C_{1}\left(k^{2}+h^{2}\right)
\end{aligned}
$$


where $C_{1}$ is a constant, which is dependent on $s_{0}, C^{*}$ and $d$ but independent on $k$ and $h$.

Now, we prove the main results (3.9a) and (3.9b) by mathematical induction. Since that $e_{i}^{j}=0$ for $i=0,1, \cdots, M, j=-N_{\tau},-N_{\tau}+1, \cdots, 0$, it is easy to check (3.9a) and (3.9b) hold for $n=0$. Suppose that (3.9a) and (3.9b) hold for $n=0,1, \cdots, m$, we will show the main results hold for $n=m+1$. First, by mean value theorem, for $i=0,1, \cdots, M$,

$$
F\left(u_{i}^{n+\frac{1}{2}-N_{\tau}}, U_{i}^{n+\frac{1}{2}-N_{\tau}}\right) e_{i}^{n+\frac{1}{2}}=f^{\prime}\left(\xi_{i}^{n}\right) e_{i}^{n+\frac{1}{2}-N_{\tau}} e_{i}^{n+\frac{1}{2}},
$$

where $\xi_{i}^{n} \in\left(u_{i}^{n+\frac{1}{2}-N_{\tau}}, U_{i}^{n+\frac{1}{2}-N_{\tau}}\right)$. Noting that (3.9a) holds for $n \leq m-1$, there exists a constant $C_{i}^{n}$ such that $f^{\prime}\left(\xi_{i}^{n}\right) \leq C_{i}^{n}$. This implies that

$$
F\left(u_{i}^{n+\frac{1}{2}-N_{\tau}}, U_{i}^{n+\frac{1}{2}-N_{\tau}}\right) e_{i}^{n+\frac{1}{2}} \leq \frac{C_{i}^{n}}{2}\left|e_{i}^{n+\frac{1}{2}-N_{\tau}}\right|^{2}+\frac{C_{i}^{n}}{2}\left|e_{i}^{n+\frac{1}{2}}\right|^{2} .
$$

Similarly, there exist constants $\widetilde{C}_{i}^{n}$ and $\bar{C}_{i}^{n}$ such that

$$
\begin{aligned}
& \left(u_{i}^{n+\frac{1}{2}-N_{\tau}}, U_{i}^{n+\frac{1}{2}-N_{\tau}}\right) \phi^{n+\frac{1}{2}} \leq \frac{\widetilde{C}_{i}^{n}}{2}\left|e_{i}^{n+\frac{1}{2}-N_{\tau}}\right|^{2}+\frac{\widetilde{C}_{i}^{n}}{2}\left|\phi^{n+\frac{1}{2}}\right|^{2}, \\
& \left(u_{i}^{n+\frac{1}{2}-N_{\tau}}, U_{i}^{n+\frac{1}{2}-N_{\tau}}\right) \psi^{n+\frac{1}{2}} \leq \frac{\bar{C}_{i}^{n}}{2}\left|e_{i}^{n+\frac{1}{2}-N_{\tau}}\right|^{2}+\frac{\bar{C}_{i}^{n}}{2}\left|\psi^{n+\frac{1}{2}}\right|^{2} .
\end{aligned}
$$

Let $n=m$ in (3.13). Substituting (3.14), (3.15a) and (3.15b) into (3.13), we have there exists a constant $C_{2}$ (independent of $h$ and $k$ ) such that

$$
\begin{aligned}
& \frac{1}{k}\left(\left\|e^{m+1}\right\|^{2}+\left|\widetilde{\phi}^{m+1}\right|^{2}+\left|\widetilde{\psi}^{m+1}\right|^{2}-\left\|e^{m}\right\|^{2}-\left|\widetilde{\phi}^{m}\right|^{2}-\left|\widetilde{\psi}^{m}\right|^{2}\right)+\left|e^{m+\frac{1}{2}}\right|_{1}^{2} \\
\leq & C_{2}\left(\left\|e^{m+1}\right\|^{2}+\left\|e^{m}\right\|^{2}+\left|\widetilde{\psi}^{m+1}\right|^{2}+\left|\widetilde{\psi}^{m}\right|^{2}+\left|\widetilde{\phi}^{m+1}\right|^{2}+\left|\widetilde{\phi}^{m}\right|^{2}+\left(k^{2}+h^{2}\right)\right),
\end{aligned}
$$

Summing up the above inequality for $m$ from 1 to $m$ and using Lemma 3.2 yields

$$
\left\|e^{m+1}\right\|^{2}+\left|\tilde{\phi}^{m+1}\right|^{2}+\left|\widetilde{\psi}^{m+1}\right|^{2}+k \sum_{i=0}^{m}\left|e^{i+\frac{1}{2}}\right|_{1}^{2} \leq C_{3}\left(k^{2}+h^{2}\right)^{2},
$$

where $C_{3}$ is a constant independent of $h$ and $k$. Moreover, by Lemma 3.3, we have

$$
\left|e^{m+1}\right|_{1} \leq k^{-1} \sqrt{T}\left(k \sum_{i=0}^{m}\left|e^{i+\frac{1}{2}}\right|_{1}^{2}\right)^{1 / 2} \leq \sqrt{T C_{3}}\left(k+k^{-1} h^{2}\right),
$$

Together with Lemma 3.1, we obtain

$$
\begin{aligned}
& \left\|U^{m+1}\right\|_{\infty}+\left|\Phi^{m+1}\right|+\left|\Psi^{m+1}\right| \leq\left\|u^{1}\right\|_{\infty}+\left|\phi^{1}\right|+\left|\psi^{1}\right|+\left\|e^{m+1}\right\|_{\infty}+\left|\widetilde{\phi}^{m+1}\right|+\left|\widetilde{\psi}^{m+1}\right| \\
\leq & \left\|u^{m+1}\right\|_{\infty}+\left|\phi^{m+1}\right|+\left|\psi^{m+1}\right|+C\left(k^{2}+h^{2}+k+k^{-1} h^{2}\right) \leq K,
\end{aligned}
$$

when $k, h$ and $k^{-1} h^{2}$ are sufficiently small. Hence, (3.9a) and (3.9b) hold for $n=m+1$. The proof is complete.

Moreover, the following stability result is imitatively obtained from the boundedness of the numerical approximations. 
Theorem 3.2. Let $\left\{U_{i}^{n}, \Phi^{n}, \Psi^{n}\right\}$ be the numerical approximations of $\left\{u\left(x_{i}, t_{n}\right), \phi\left(t_{n}\right), \psi\left(t_{n}\right)\right\}$. Then, the proposed numerical schemes are stable when $k, h$ and $k^{-1} h^{2}$ are sufficiently small.

\section{Numerical experiments}

In this section, we will illustrate the efficiency of LABCs and, confirm theoretical results of our numerical scheme, and also test the influence of the delay on the traveling wave.

\subsection{Effectiveness of the proposed method}

Example 4.1. We show an accuracy test for the Nicholson's blowflies equation

$$
u_{t}-D u_{x x}=-d u(x, t)+b u(x, t-\tau) \exp (-a u(x, t-\tau)), \quad(x, t) \in \mathbb{R} \times \mathbb{R}^{+}
$$

with the initial condition

$$
u(x, t)=\frac{1}{\sqrt{2 \pi}} \exp \left(-\frac{x^{2}}{2}\right), \quad(x, t) \in \mathbb{R} \times[-\tau, 0] .
$$

We take the parameters by $D=1, d=2, b=1, s_{0}=5$ and $\tau=1$. We set stepsize $h=k$, and solve the problem on the domain $[-6,6] \times[0,4]$ with LABCs $(2.16 \mathrm{c})$ and $(2.16 \mathrm{~d})$. Since that the exact solutions of the problem are unknown, the reference solutions are computed by using stepsizes $h=k=10^{-4}$ on the computational domain $[-10,10]$. In Table 1, we list the errors and the convergence order. As we can see, these numerical results confirm the effectiveness of our LABCs and numerical scheme.

Example 4.2. We further show an accuracy test for the Mackey-Glass equation

$$
u_{t}-D u_{x x}=-d u(x, t)+\frac{b u(x, t-\tau)}{1+u^{2}(x, t-\tau)}, \quad(x, t) \in \mathbb{R} \times \mathbb{R}^{+}
$$

with the initial value

$$
u(x, t)=\exp \left(-x^{2}\right), \quad(x, t) \in \mathbb{R} \times[-\tau, 0] .
$$

Table 1: The errors at different time and corresponding orders for Eq. (4.1)

\begin{tabular}{||c|cc|cc|cc|cc||}
\hline & \multicolumn{2}{|c|}{$t=1$} & \multicolumn{2}{c|}{$t=2$} & \multicolumn{2}{c||}{$t=3$} & \multicolumn{2}{c||}{$t=4$} \\
$k$ & error & order & error & order & error & order & error & order \\
\hline 0.2 & $1.00 \mathrm{E}-3$ & - & $3.44 \mathrm{E}-4$ & - & $1.23 \mathrm{E}-4$ & - & $7.82 \mathrm{E}-5$ & - \\
0.1 & $2.50 \mathrm{E}-4$ & 2.00 & $8.52 \mathrm{E}-4$ & 2.01 & $3.10 \mathrm{E}-5$ & 1.99 & $1.95 \mathrm{E}-5$ & 2.00 \\
0.05 & $6.23 \mathrm{E}-5$ & 2.00 & $2.12 \mathrm{E}-5$ & 2.01 & $7.74 \mathrm{E}-6$ & 2.00 & $4.84 \mathrm{E}-6$ & 2.01 \\
0.025 & $1.54 \mathrm{E}-5$ & 2.02 & $5.23 \mathrm{E}-5$ & 2.01 & $1.91 \mathrm{E}-5$ & 2.01 & $1.19 \mathrm{E}-6$ & 2.01 \\
\hline
\end{tabular}


Table 2: The errors at different time and corresponding orders for Eq. (4.3).

\begin{tabular}{||c|cc|cc|cc|cc||}
\hline & \multicolumn{2}{|c|}{$t=1$} & \multicolumn{2}{c|}{$t=2$} & \multicolumn{2}{c||}{$t=3$} & \multicolumn{2}{c||}{$t=4$} \\
$k$ & error & order & error & order & error & order & error & order \\
\hline 0.2 & $1.55 \mathrm{E}-3$ & - & $8.03 \mathrm{E}-4$ & - & $5.37 \mathrm{E}-4$ & - & $4.45 \mathrm{E}-5$ & - \\
0.1 & $3.82 \mathrm{E}-4$ & 2.02 & $1.95 \mathrm{E}-4$ & 2.04 & $1.28 \mathrm{E}-4$ & 2.06 & $1.08 \mathrm{E}-4$ & 2.04 \\
0.05 & $9.56 \mathrm{E}-5$ & 2.00 & $4.82 \mathrm{E}-5$ & 2.02 & $3.17 \mathrm{E}-5$ & 2.01 & $2.67 \mathrm{E}-5$ & 2.02 \\
0.025 & $2.36 \mathrm{E}-5$ & 2.01 & $1.19 \mathrm{E}-5$ & 2.02 & $7.80 \mathrm{E}-6$ & 2.02 & $6.57 \mathrm{E}-6$ & 2.02 \\
\hline
\end{tabular}

We take the parameters by $D=1, d=2, b=2, m=2, s_{0}=10$ and $\tau=1$. Again, we set stepsize $h=k$ and solve the problem on the domain $[-6,6] \times[0,4]$ with our LABCs $(2.16 \mathrm{c})$ and (2.16d). The reference solutions are obtained by taking stepsizes $h=k=10^{-4}$. The errors at different time and convergence orders are shown in Table 2. Again, the convergence order is consistent with the theoretical analysis.

\subsection{Application of the ABCs and the numerical schemes}

In this subsection, we investigate the influence of the delay on the the traveling wave.

Example 4.3. Consider the Nicholson's blowflies equation (4.1) with the initial condition

$$
u(x, t)=\frac{\ln p-\ln d}{a\left(1+e^{-5 x}\right)}, \quad(x, t) \in \mathbb{R} \times[-\tau, 0],
$$

where $p>d$. Clearly,

$$
u_{-}:=\lim _{x \rightarrow-\infty} u(x, t)=0, \quad u_{+}:=\lim _{x \rightarrow+\infty} u(x, t)=\frac{1}{a} \ln \frac{p}{d} .
$$

These are two equilibria of the problem (4.1). Next, we are interested in investigating the influence of the delay on the traveling wave. We set the parameter $D=1, d=1$, $a=1, s_{0}=6$ and $p=10$, and then solve the problem on the computational domain $[-20,10] \times[0, T]$ with the proposed nonlinear ABCs. The numerical results, which are obtained by taking stepsizes $k=h=0.1$, are shown in Figs. 1-6. Fig. 1 and Fig. 2 indicate that the numerical solutions behave like some certain monotone traveling waves, when the delay is small (for example, $\tau=0.1$ ). Fig. 3 and Fig. 4 indicate that the numerical solutions behave like some oscillatory traveling waves, when the delay is big (for example, $\tau=1$ ). Fig. 5 and Fig. 6 indicate that the numerical solutions behave like some chaotic oscillations, when the delay becomes lager (for example, $\tau=5$ ). These numerical results not only suggest that the delay term has an important impact on the dynamical behaviors of the model, but also numerically illustrate some theoretical results or conjectures on the traveling wave. 


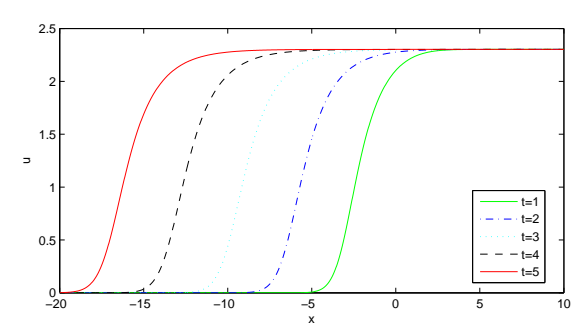

Figure 1: Numerical solutions at different time levels.

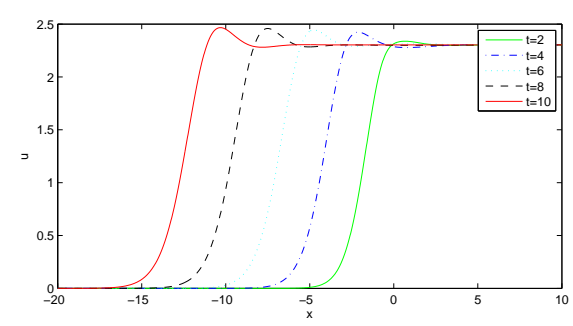

Figure 3: Numerical solutions at different time levels.

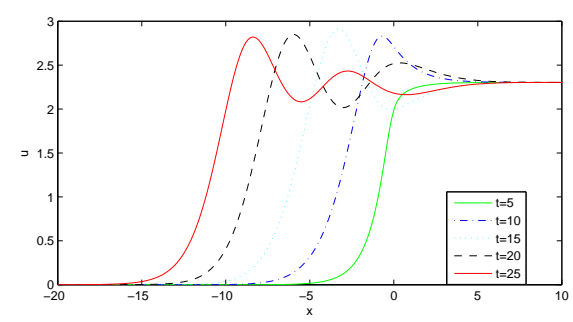

Figure 5: Numerical solutions at different time levels.

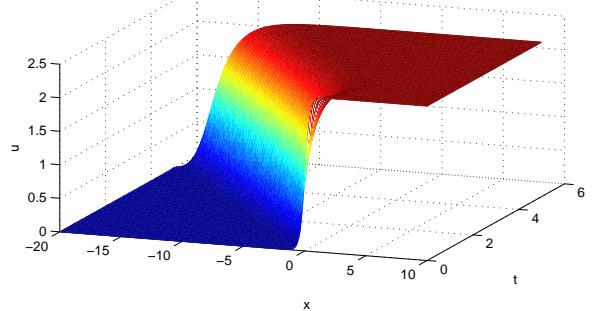

Figure 2: Numerical solutions with $\tau=0.1$.

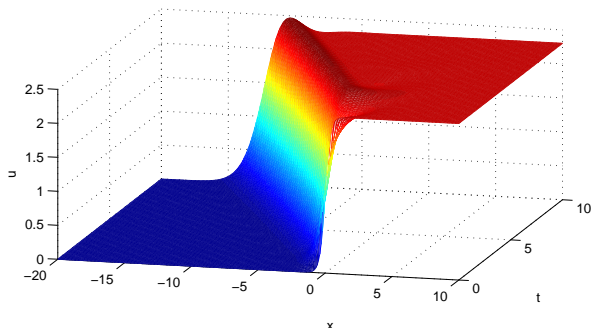

Figure 4: Numerical solutions with $\tau=1$.

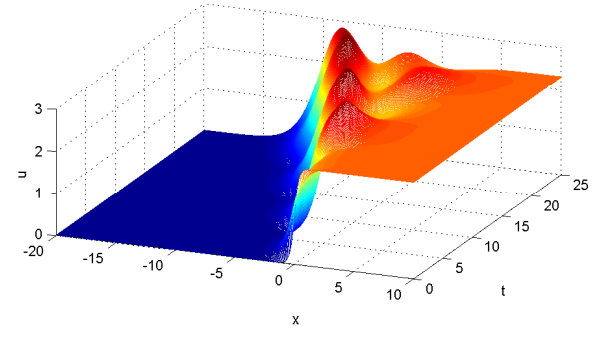

Figure 6: Numerical solutions with $\tau=5$.

Example 4.4. Consider the Mackey-Glass equation (4.3) with the initial condition

$$
u(x, t)=\frac{\sqrt{P / d-1}}{\left(1+e^{-5 x}\right)}, \quad(x, t) \in \mathbb{R} \times[-\tau, 0],
$$

where $p>d$. Clearly,

$$
u_{-}:=\lim _{x \rightarrow-\infty} u(x, t)=0, \quad u_{+}:=\lim _{x \rightarrow+\infty} u(x, t)=\sqrt{P / d-1} .
$$

These two limitations are equilibria of problem (4.3). Next, we are concerned with the influence of the delay on the traveling wave. We still set the parameter $D=1, d=1$, $s_{0}=6$ and $p=10$, and then solve the problem on the computational domain $[-20,10] \times$ $[0, T]$ with the proposed nonlinear ABCs. We also plot the numerical results in Figs. 712 , where the numerical results are obtained by taking stepsizes $k=h=0.1$. It can be seen clearly from these figures that when the delay term becomes larger and larger, the numerical solutions become more and more complicated. In these figures, they behave 


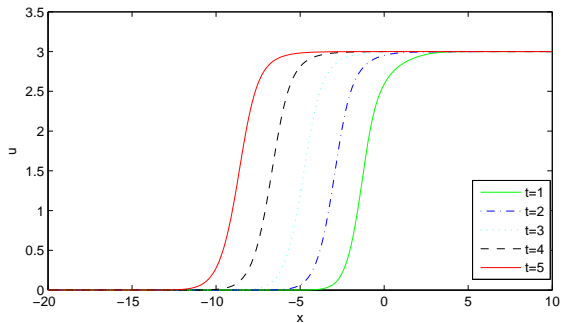

Figure 7: Numerical solutions at different time levels.

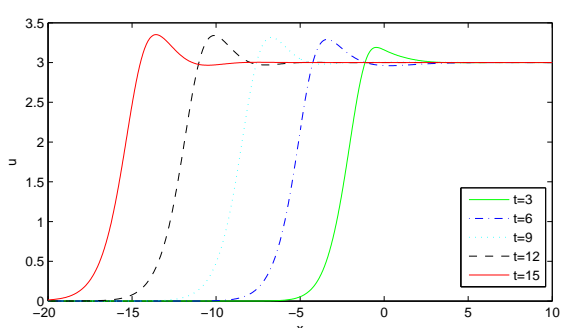

Figure 9: Numerical solutions at different time levels.

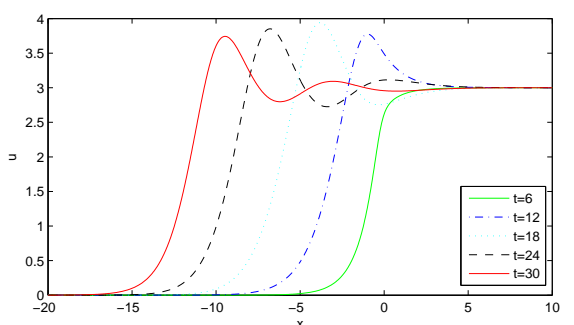

Figure 11: Numerical solutions at different time levels.

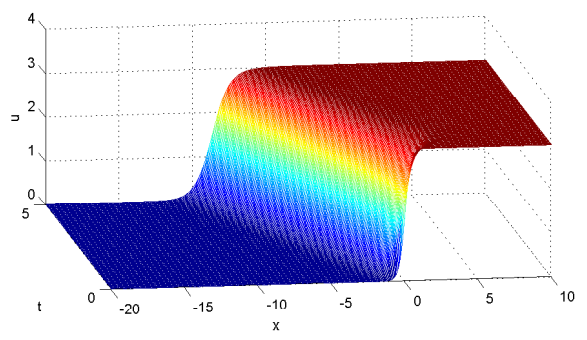

Figure 8: Numerical solutions with $\tau=0.1$.

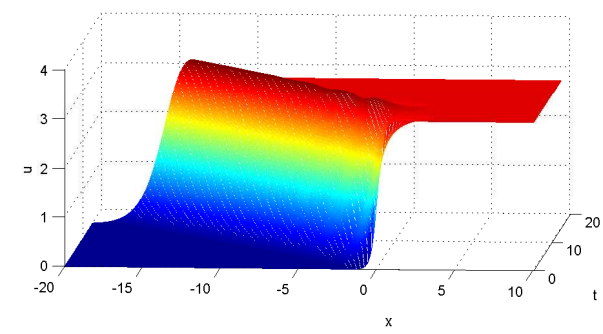

Figure 10: Numerical solutions with $\tau=1$.

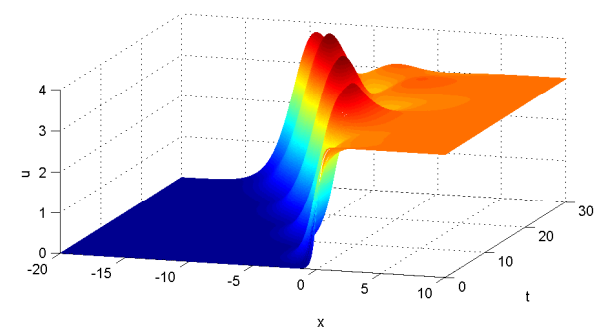

Figure 12: Numerical solutions with $\tau=5$.

like certain monotone traveling waves, oscillatory traveling waves and chaotic oscillations, respectively. These illustrate that the delay term have an important impact on the traveling wave. To the best of our knowledge, these interesting dynamical behaviors for problem (4.3) have never been well investigated. In the present paper, we give some numerical findings on these dynamical behaviors.

\section{Conclusions and discussions}

In this study, we first constructed efficient local absorbing boundary conditions to reformulate the reaction-diffusion equations with time delay on unbounded domain into a reduced problem on a bounded domain. After that, we proposed a linearized finite $\mathrm{d}$ ifference method to solve the reduced problem. Numerical experiments on two typical biological models are given to demonstrate the efficiency of our LABCs and numerical 
scheme. Most importantly, we investigated the influence of the delay term on the dynamical behaviors of traveling waves, and found that the traveling wave can behave like a certain monotone traveling wave, oscillatory traveling wave and an chaotic oscillation, respectively, under different time delay. A direct extension of this work is to consider the multi-dimensional case and some time-fractional models $[18,21]$ in the future.

Acknowledgments This work is supported in part by the National Natural Science Foundation of China ( Grant Nos. 11771162, 11771035,11571027, 91430216 and U1530401), Beijing Nova Program (No. Z151100003150140), and Scientific Research Project of Beijing Educational Committee (No. KM201510005032).

\section{References}

[1] P. R. Agarwal, L. Berezansky, E. Braverman and A. Domoshnitsky, Nonoscillation Theory of Functional Differential Equations with Applications, Springer, New York, 2012.

[2] X. Antoine, C. Besse and S. Descombes, Artificial boundary conditions for one-dimensional cubic nonlinear Schrödinger equations, SIAM J. Numer. Anal., 43 (2006), pp. 2272-2293.

[3] L. BEREZANSKY, E. BRAVERMAN AND L. IDELs, Nicholson's blowflies differential equations revisited: Main results and open problems, Appl. Math. Model., 34 (2010), pp. 1405-1417.

[4] H. Brunner, H. Li And X. Wu, Numerical Solution of Blow-Up Problems for Nonlinear Wave Equations on Unbounded Domains, Commu. Comput. Phys., 14 (2013), pp. 574-598.

[5] I. Chern, M. MeI, X. YANG AND Q. ZhANG, Stability of non-monotone critical traveling waves for reaction-diffusion equations with time-delay, J. Diff. Equ., 259 (2015), pp. 1503-1541.

[6] D. Givoli, High-order local non-reflecting boundary conditions: A review, Wave Motion, 39 (2004), pp. 319-326.

[7] T. Hagstrom, New results on absorbing layers and radiation boundary conditions, Lect. Notes Comp. Sci., (31) 2003, pp. 1-42.

[8] H. HAn AND X. WU, Artificial Boundary Method, Spring-Verlag and Tsinghua Unversity Press, Berlin Heidelberg and Beijing, 2013.

[9] C. Huang And S. Vandewalle, An Analysis of Delay-Dependent Stability for Ordinary and Partial Differential Equations with Fixed and Distributed Delays, SIAM J. Sci. Comput., 25 (2004), pp. 1608-1632.

[10] J. G. Heywood and R. RanNacher, Finite element approximation of the nonstationary Navier Stokes problem IV: Error analysis for second-order time discretization, SIAM J. Numer. Anal., 27 (1990), pp. 353-384.

[11] Z. JACKIEWICZ AND B. ZuBiK-Kowal, Spectral collocation and waveform relaxation methods for nonlinear delay partial differential equations, Appl. Numer. Math., 56 (2006), pp. 433-443.

[12] T. Hagstrom and H. B. Keller, Asymptotic boundary conditions and numerical methods for nonlinear elliptic problems on unbounded domains, Math. Comp., 48 (1987), pp. 449-470.

[13] T. Hagstrom and H. B. Keller, The Numerical Calculation of Traveling Wave Solutions of Nonlinear Parabolic Equations, SIAM J. Sci. Stat. Comp., 7(3) (1986), pp. 978-988.

[14] L. A. KHAN AND P. L. F. LiU, Numerical analyses of operator-splitting algorithms for the twodimensional advection-diffusion equation, Comput. Meth. Appl. Mech. Eng., 152 (1998), pp. 337-359.

[15] D. LI AND J. WANG, Unconditionally optimal error analysis of Crank-Nicolson Galerkin FEMs for a strongly nonlinear parabolic system, J. Sci. Comput., 72 (2017), pp. 892-915. 
[16] D. LI, C. ZHANG AND H. QIN, LDG method for reaction-diffusion dynamical systems with time delay, Appl. Math. Comput., 217 (2011), pp. 9173-9181.

[17] D. Li AND C. ZhANG, Superconvergence of a discontinuous Galerkin methods for first-order linear delay differential equations, J. Comp. Math., 29 (2011), pp. 574-588.

[18] D. LI, C. ZHANG AND Z. ZHANG, Unconditionally optimal error estimates of a linearized Galerkin method for nonlinear time fractional reaction-subdiffusion equations, J. Sci. Comput., 76 (2018), pp. 848-866.

[19] C. Lin, C. Lin, Y. Lin And M. MeI, Exponential stability of nonmonotone traveling waves for Nicholson's blowflies equations, SIAM J. Appl. Math., 46 (2014), pp. 1053-1084.

[20] D. LI AND J. ZHANG, Efficient implementation to numerically solve the nonlinear time fractional parabolic problems on unbounded spatial domain, J. Comput. Phys., 322 (2016), pp. 415-428.

[21] D. LI, J. WANG AND J. ZHANG, Unconditionally convergent $L^{1}$-Galerkin FEMs for nonlinear timefractional Schrödinger equations, SIAM. J. Sci. Comput., 39(6) (2017), pp. A3067-A3088.

[22] C. Liang AND X. XIANG, Convergence of an anisotropic perfectly matched layer method for Helmholtz scattering problems, Numer. Math. TMA., 9 (2016), pp. 358-382.

[23] M. MeI, C. Ou AND X. Zhaо, Global stability of monostable traveling waves for nonlocal timedelayed reacton-diffusion equations, SIAM J. Appl. Math., 42 (2010), pp. 2762-2790.

[24] G. StRAnG, On the construction and comparison of difference schemes, SIAM J. Numer. Anal., 5 (1968), pp. 506-517.

[25] S. H. Strogatz, Nonlinear dynamics: death by delay, Nature, 394 (1998), pp. 316-317.

[26] Z. Sun, X. Wu, Z. ZHANG AND D. WANG, A linearized difference scheme for semilinear parabolic equations with nonlinear absorbing boundary conditions, Appl. Math. Comp., 218 (2012), pp. 5187-5201.

[27] Z. Sun And Z. Zhang, A linearized compact difference scheme for a class of nonlinear delay partial differential equations, Appl. Math. Model., 37 (2013), pp. 742-752.

[28] H. Song, K. Zhang And Y. LI, Finite Element and Discontinuous Galerkin Methods with Perfect Matched Layers for American Options, Numer. Math. Theory Methods Appl., 10(4) (2017), pp. 829-851.

[29] S. V. Tsynkov, Numerical solution of problems on unbounded domains: A review, Appl. Numer. Math., 27 (1998), pp. 465-532.

[30] J. WANG, A new error analysis of Crank-Nicolson Galerkin FEMs for a generalized nonlinear Schrödinger equation, J Sci. Comput. 60 (2014), pp. 390-407.

[31] J. H. Wu, Theory and Application of Partial Functional Differential Equation, Springer, 1996.

[32] X. Wu AND J. Zhang, Artificial boundary method for two-dimensional Burgers' equation, Comput. Math. Appl., 56 (2008), pp. 242-256.

[33] X. WU AND J. ZHANG, Higher-order local absorbing boundary conditions for heat equations in unbounded domain, J. Comput. Math., 29 (2011), pp. 74-90.

[34] X. WU AND W. ZHENG, An adaptive perfectly matched layer method for multiple cavity scattering problems, Commun. Comput. Phys., 19 (2016), pp. 534-íc558.

[35] Z. Xu, H. HAN AND X. Wu, Numerical method for the deterministic Kardar-Parisi-Zhang equation in unbounded domains, Commun. Comput. Phys., 1 (2006), pp. 479-493.

[36] Z. XU, H. HAN AND X. WU, Adaptive absorbing boundary conditions for Schrödinger-type equations: application to nonlinear and multi-dimensional problems, J. Comput. Phys., 225 (2007), pp. 1577-1589.

[37] G. Zhang AND A. XIAO, Stability and convergence analysis of implicit-explicit one-leg methods for stiff delay differential equations, Int. J. Comput. Math., 93 (2016), pp. 1964-1983.

[38] Q. ZHANG AND C. ZHANG, A compact difference scheme combined with extrapolation techniques for solving a class of neutral delay parabolic differential equations, Appl. Math. Lett., 26 (2013), 
pp. 306-312.

[39] D. Li, C. Zhang AND J. Wen, A note on compact finite difference method for reaction-diffustion equations with delay, Appl. Math. Model., 39 (2015), pp. 1749-1754.

[40] J. ZHANG, Z. XU AND X. WU, Unified approach to split absorbing boundary conditions for nonlinear Schrödinger equations, Phys. Rev. E, (3)(78) 2008, 026709.

[41] J. ZhANG, Z. XU AND X. Wu, Unified approach to split absorbing boundary conditions for nonlinear Schrödinger equations: Two dimensional case, Phys. Rev. E, (3)(79) 2009, 046711.

[42] W. Zhang, J. Yang, J. Zhang and Q. Du, Artificial Boundary Conditions for Nonlocal Heat Equations on Unbounded Domain, Commu. Comput. Phys., 21 (2017), pp. 16-39.

[43] C. ZHENG, Exact nonreflecting boundary conditions for one-dimensional cubic nonlinear Schrödinger equations, J. Comput. Phys., 215 (2006), pp. 552-565.

[44] C. Zheng, Numerical solution to the sine-Gordon equation defined on the whole real axis, SIAM J. Sci. Comput., 29 (2007), pp. 2494-2506.

[45] W. Zou, ET AL., Restoration of rhythmicity in diffusively coupled dynamical networks, Nature Commun., 6 (2015), pp. 7709. 